\title{
Endoscopic sleeve gastroplasty: the learning curve
}

\section{(ㄷ) $(>)$}

\author{
Authors \\ Christine Hill ${ }^{1}$, Mohamad El Zein ${ }^{2}$, Abhishek Agnihotri ${ }^{3}$, Margo Dunlap ${ }^{2}$, Angela Chang ${ }^{2}$, Alison Agrawal ${ }^{2}$, Sindhu \\ Barola$^{2}$, Saowanee Ngamruengphong ${ }^{2}$, Yen-I Chen², Anthony N. Kalloo ${ }^{2}$, Mouen A. Khashab², Vivek Kumbhari²
}

Institutions

1 Diversity Summer Internship Program, Johns Hopkins Bloomberg School of Public Health, Baltmore, Maryland, United States

2 Division of Gastroenterology and Hepatology, Department of Medicine, The Johns Hopkins Medical Institutions, Baltimore, Maryland, United States

3 Department of Medicine, Johns Hopkins University of Medicine, Baltimore, Maryland, United States

submitted 2.5.2017

accepted after revision 16.6.2017

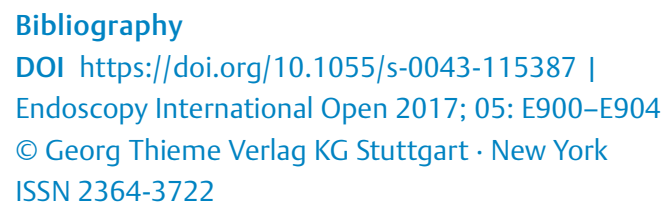

Corresponding author

Vivek Kumbhari, M.B. Ch.B., Assistant Professor of Medicine, Division of Gastroenterology and Hepatology, Director of Bariatric Endoscopy, Johns Hopkins Medical Institutions, 4940 Eastern Avenue, AA Building, 3rd floor Phone: Baltimore, MD 21224

Fax: +1 410 550-7861

vkumbhari@gmail.com

\section{ABSTRACT}

Background and study aims Endoscopic sleeve gastroplasty (ESG) is gaining traction as a minimally invasive bar- iatric treatment. Concern that the learning curve may be slow, even among those proficient in endoscopic suturing, is a barrier to widespread implementation of the procedure. Therefore, we aimed to define the learning curve for ESG in a single endoscopist experienced in endoscopic suturing who participated in a 1-day ESG training program.

Patients and methods Consecutive patients who underwent ESG between February 2016 and November 2016 were included. The performing endoscopist, who is proficient in endoscopic suturing for non-ESG procedures, participated in a 1-day ESG training session before offering ESG to patients. The outcome measurements were length of procedure (LOP) and number of plications per procedure. Nonlinear regression was used to determine the learning plateau and calculate the learning rate.

Results Twenty-one consecutive patients (8 males), with mean age $47.7 \pm 11.2$ years and mean body mass index $41.8 \pm 8.5 \mathrm{~kg} / \mathrm{m}^{2}$ underwent ESG. LOP decreased significantly across consecutive procedures, with a learning plateau at 101.5 minutes and a learning rate of 7 cases $(P=0.04)$. The number of plications per procedure also decreased significantly across consecutive procedures, with a plateau at 8 sutures and a learning rate of 9 cases $(P<0.001)$. Further, the average time per plication decreased significantly with consecutive procedures, reaching a plateau at 9 procedures $(P<0.001)$.

Conclusions Endoscopists experienced in endoscopic suturing are expected to achieve a reduction in LOP and number of plications per procedure in successive cases, with progress plateauing at 7 and 9 cases, respectively.

\section{Introduction}

Endoscopic sleeve gastroplasty (ESG) has recently emerged as a safe and effective minimally invasive procedure for treatment of obesity [1-5]. ESG has been associated with weight loss comparable to laparoscopic adjustable band surgery, with individual centers reporting excess weight loss (EWL) over $50 \%$ at 1 year post-procedure $[1,5,6]$. Significant weight loss coupled with a relative lack of adverse events, short recovery period, and cost-effectiveness makes ESG ideal for treatment of obesi- ty in patients that do not qualify for, or object to, bariatric surgery $[1,2,4]$.

ESG reduces stomach volume using a full-thickness endoscopic suturing system, in a mechanism that mimics that of laproscopic sleeve gastrectomy. This results in the gastric cavity forming a tubular structure along the lesser curvature, with the greater curvature closed off by plications from the gastroesophogeal junction to the prepyloric antrum $[3,4,7]$. Weight loss has been found to be durable at 1-year follow up [5]. Further, the anatomy has the potential to be reversed [3]. 
For widespread use of any endoscopic therapy, the procedure must be effective, safe, and able to be performed by a large number of providers. Currently, many endoscopists resist performing ESG because of the belief that it requires time-consuming training and has a slow learning curve. However, the learning curve for ESG has yet to be defined. Therefore, we aimed to define the learning curve for ESG, specifically, following a 1-day training program undertaken by an endoscopist experienced in suturing.

\section{Patients and methods}

This retrospective study was approved by our Institutional Review Board for Human Research and complied with Health Insurance Portability and Accountability Act (HIPAA) regulations.

The performing endoscopist achieved proficiency in endoscopic suturing for non-ESG procedures prior to ESG training. Examples of these procedures include but are not limited to suturing for covered stent fixation, closure of endoscopic submucosal dissection sites, and transoral gastric outlet reduction. The endoscopist performed $>20$ of these procedures before beginning ESG training. ESG training was limited to 1 day, and consisted of a half-day didactic lecture followed by 1 proctored ESG procedure in a porcine explant stomach. The session was led by a group of physicians who had been performing ESG for more than 1 year. Proctoring for the explant ESG was 1-on-1, while the didactic portion of the training was not. The lecture was classroom-style, but incorporated the ability to ask questions. The specific topics covered in the lecture included evolution of the procedure, demonstration of optimal suturing patterns, technical pearls, and troubleshooting tips for commonly encountered problems. Notably, a complete ESG procedure was not shown from start to finish in the training or in other available resources. Outside of this formal training, the endoscopist read articles about ESG and watched videos of portions of the procedure, but did not have the ability to see the entire procedure before performing it.

Upon completion of the 1-day training session, the endoscopist began offering ESG to patients. The first ESG procedure performed on a patient occurred 1 week after the training session. All consecutive patients who underwent ESG by the single endoscopist (V.K.) between the commencement of the program, in February 2016, and the completion of the study, in November 2016, were included in the learning curve analysis. All ESG procedures took place at the Johns Hopkins Medical Institutions (Baltimore, Maryland, United State). Further, the anesthesia and nursing teams were consistent throughout each procedure. Members of the nursing team were familiar with the OverStitch device but they were naïve to ESG prior to commencement of the program.

Prior to ESG, all patients were counseled on the spectrum of therapies available to treat obesity, including diet and lifestyle, pharmaceutical treatment, endoscopic bariatric therapies, and bariatric surgery. The decision to perform ESG was made only after a patient demonstrated an understanding of all available treatment options. Unless contraindications appeared to be likely based on clinical history and physical examination, no endoscopic assessment was performed prior to ESG. Contraindications for ESG include prior gastric surgery, gastric ulceration, acute gastritis, anticoagulation, pregnancy, and psychiatric disorders that pose a substantial risk of interfering with the patient's ability to follow post-procedural instructions or make the recommended lifestyle adjustments post-ESG [1,4, 5]. Patients were given an electronic prescription 2 weeks prior to the procedure, which included omeprazole $40 \mathrm{mg}$ qD to be taken 1 week pre-ESG and for 6 weeks post-ESG, emend (aprepitant) $125 \mathrm{mg}$ to be taken 3 hours prior to ESG, emend (Aprepitant) $80 \mathrm{mg}$ qD to be taken the day after the procedure, hyoscyamine (Levsin) $0.125 \mathrm{mg} 1 \mathrm{tab}$ q $6 \mathrm{~h}$ as needed for cramping post-ESG, and ondansetron (Zofran) $4 \mathrm{mg}$ sublingual tab 1 tab q $6 \mathrm{~h}$ as needed for nausea post-ESG. On the day preceding the procedure, a liquid diet was prescribed with no intake permitted after midnight.

ESG was completed as a day case, with no overnight hospitalization required. The patient was positioned in the left lateral position. General anesthesia was administered via inhalation gas throughout the procedure. Fentanyl 100 mcg, ondansetron $8 \mathrm{mg}$ and dexamethasone $8 \mathrm{mg}$ were delivered intravenously (IV) within a predicted 30 minutes from completion of the procedure. During the pre-procedure, intra-procedure and postprocedural time, $2 \mathrm{~L}$ of IV fluids were administered.

All ESG procedures were performed as described by LopezNava et al. [4, 5, 8] and Sharaiha et al. [2]. The suture line was first marked with argon plasma coagulation (APC) along the anterior wall, greater curvature, and posterior wall. Using a full-thickness endoscopic suturing system (OverStitch; Apollo Endosurgery, Austin, Texas, USA) the sutures were placed distally to proximally (from the incisura angularis to the fundus) in a triangular pattern from the anterior wall to the greater curvature to the posterior wall. For the second row of plications, the process was repeated in the opposite direction using the argon marks to guide the endoscopist's orientation within the stomach. Importantly, full-thickness bites were taken between the guidelines on the anterior wall and the greater curvature, and between the posterior wall and the greater curvature, in order to avoid the formation of two long gastric pockets. Generally, 6 to 9 bites per suture were performed. On completion of the suture pattern, the $T$ tag was released and the stitches were then brought together to form a plication using a cinching device [9]. The suture line was brought proximally until within $1 \mathrm{~cm}$ of the gastroesophageal junction on the lesser curvature aspect. The fundus (towards the proximal body aspect) was sutured until the endoscope began to retroflex. Therefore, a small fundal pouch (approximately $15 \mathrm{~mL}$ in capacity) remained at each procedure as desired. On completion of the final plication, a quick endoscopy without the OverStitch attachment was performed to ensure ESG had an optimal appearance and absence of bleeding ( $>$ Fig. 1 ).

Post-procedure, patients were instructed to follow a liquid diet for 3 days before transitioning to smooth, pureed foods as tolerated. Successive transitions from pureed foods to soft foods and then to solid foods occurred in 2-week intervals. Medications were also prescribed to ease post-procedural symptoms of nausea, heartburn, cramping and pain. The most 


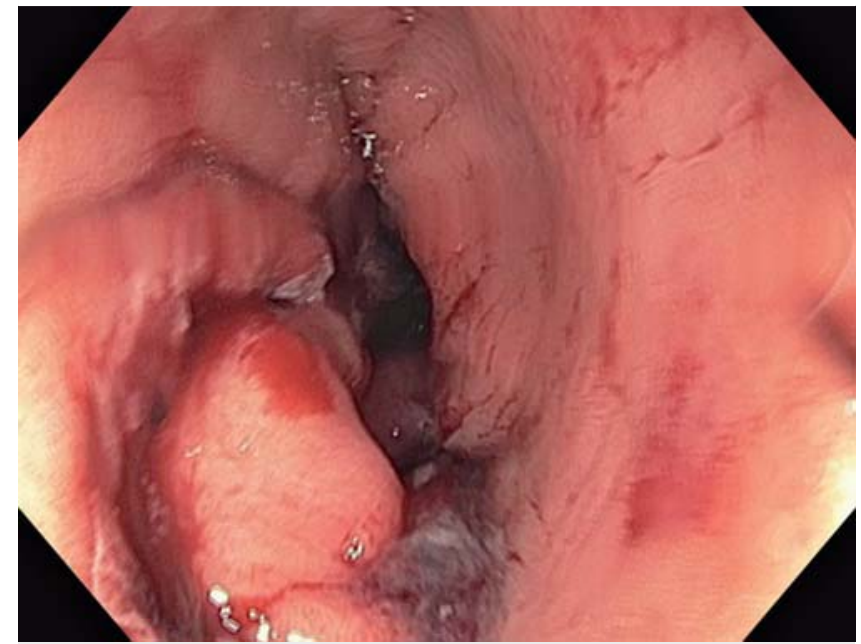

Fig. 1 Endoscopic view demonstrating gastric sleeve upon completion of the procedure.

common post-placement symptoms were nausea and pain, making dehydration a post-procedural concern.

All patients were enrolled in a comprehensive ancillary program, focused on helping them make positive dietary and lifestyle changes for a minimum of 12 months post-ESG. This program consists of routine treatment from a multidisciplinary weight loss team, composed of a gastroenterologist with expertise in weight management, a registered dietician, an exercise physiologist, and a behavioral psychologist (The Johns Hopkins Weight Management Center, Baltimore, MD).

\section{Statistical analysis}

Results are reported as mean \pm standard deviation (SD) for continuous data and as frequencies and proportions for categorical data. Continuous variables, namely pre-procedure and postprocedure body mass index (BMI) and excess body weight, were compared with paired $t$ tests. To define the "learning curve," nonlinear regression was used to fit an inverse curve, with case number as the independent variable and procedure time as the dependent variable. This statistical method yields an estimate of a (asymptote) and b (slope), as described by Feldman et al. [10]. The "learning plateau" is then defined as the procedure time at the asymptote of the learning curve (i.e., the theoretical best score that a subject could achieve with infinite practice). The "learning rate" is defined as the number of trials required to reach $90 \%$ of potential (i.e., the speed at which the subject acclimates to the task). When procedure time $=a+0.1 \times($ slowest individual procedure time $-a)$, then learning rate case number $=10 \times b /($ slowest time $-a)$. The outcome measurements were length of procedure (LOP), number of plications per procedure and average time per plication per procedure. The LOP was defined as time from initial endoscope entry into the mouth to final endoscope withdrawal. Data sets were compiled using Microsoft Excel, and all statistical analyses were performed using SPSS version 21 (SPSS Inc, Chicago, IL, USA).

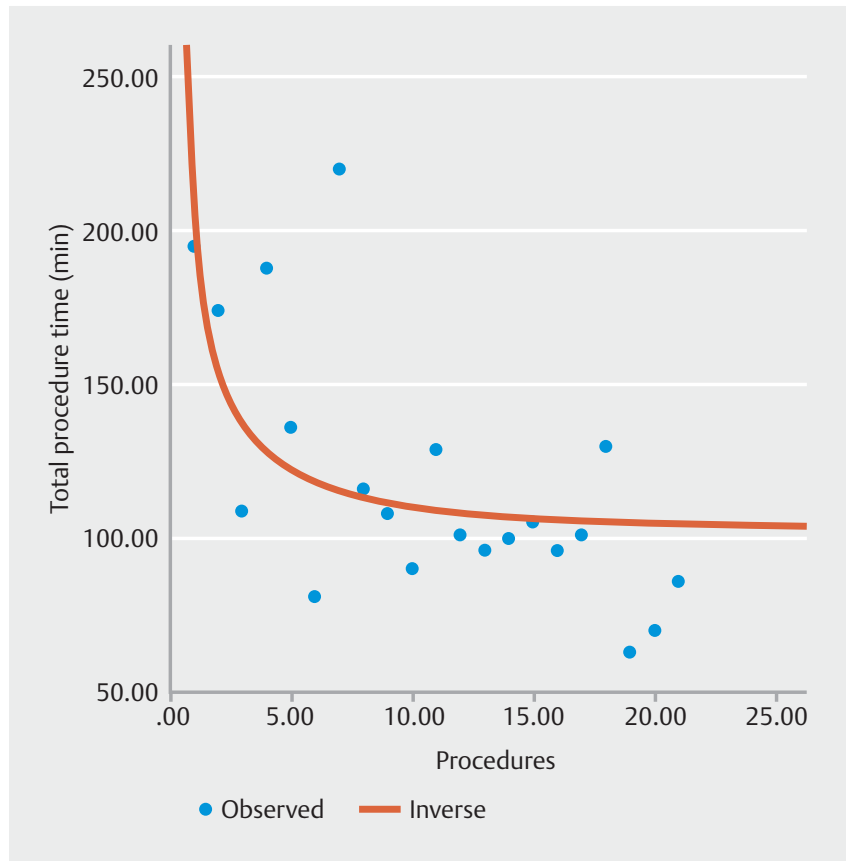

- Fig. 2 Total procedure time significantly decreased over the course of consecutive endoscopic sleeve gastroplasty procedures, with a learning plateau at 101.5 minutes and a learning rate of 7 cases $(P=0.04)$.

\section{Results}

Twenty-one consecutive patients ( 8 males) underwent ESG by the single endoscopist (V.K.) between the commencement of the program, in February of 2016, and the end of the study period, in November 2016. The mean age was $47.7 \pm 11.2$ years (range 29-73), and the mean initial BMI was $41.8 \pm 8.5 \mathrm{~kg} / \mathrm{m}^{2}$ (range $33-69$ ).

ESG was performed with technical success in all (100\%) patients. All patients had general anesthesia successfully. Bleeding was encountered on occasion with bites, but in all cases, bleeding ceased once the plication was complete.

Median LOP was 105 minutes (range 63-220). Total procedure time decreased significantly over the course of consecutive ESG procedures, with a learning plateau at 101.5 minutes and a learning rate of 7 cases $(P=0.04)$ ( $\triangleright$ Fig. 2 ). The total number of plications performed per ESG procedure also decreased significantly across the consecutive procedures. The median number of plications performed per ESG procedure was 9 (range $6-4$ ). The learning plateau was reached at 8 sutures and the learning rate was 9 cases $(P<0.001)$ ( $\mathbf{F i g . 3}$ ). Further, the average time per plication decreased significantly with consecutive procedures. The median average time per plication was 13.125 minutes (range $8.1-24.4$ ) ( $\triangleright$ Fig.4). The average time per plication reached a plateau at a rate of 9 procedures $(P<0.001)$.

One patient (4.8\%) was lost to follow-up. Thirty-day postprocedure follow-up data on the remaining 20 patients showed a mean reduction in BMI of $3.2 \pm 1.4 \mathrm{~kg} / \mathrm{m}^{2}(P<0.001)$ and a mean weight loss of $23.6 \pm 8.9 \mathrm{lb}(P<0.001)$. The immediate 


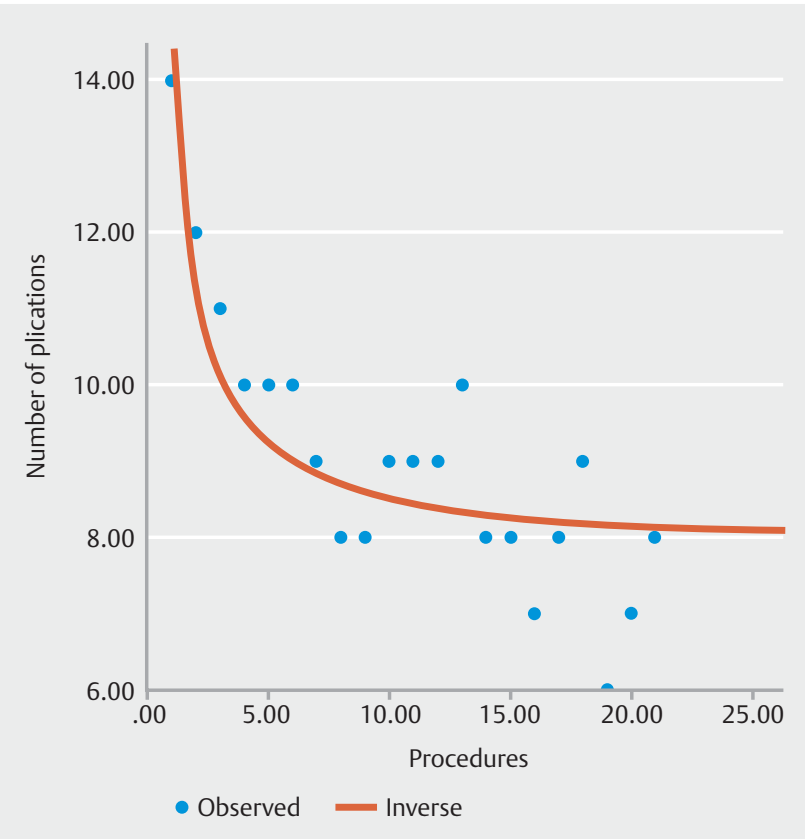

- Fig. 3 Number of plications per procedure significantly decreased over the course of consecutive endoscopic sleeve gastroplasty procedures, with a learning plateau at 8 sutures and a learning rate of 9 cases $(P<0.001)$.

readmission rate was $0 \%$, and the 30 -day readmission rate was $4.8 \%$. No immediate post-procedural complications occurred. No symptoms of gastroesophageal reflux disease (GERD) were present at 30-day follow up. One patient experienced an AE of mild severity, as classified according to the ASGE Lexicon by Cotton et al. [11]. That patient recovered well post-procedure, before presenting with abdominal pain and vomiting 12 days after ESG. The patient was diagnosed with perigastric fluid collection, which was responsive to antibiotics. Treatment required readmission, with a length of stay of 1 day. This event is discussed further by Barola et al. [7] No patients underwent a repeat procedure.

\section{Discussion}

Results of this analysis indicate that the LOP, number of plications per procedure, and average time spent per plication per procedure decreased significantly with the number of procedures performed. The decrease in time spent per plication and the decrease in total LOP are straightforward indicators of efficiency, however, the decrease in the number of plications used per procedure appears to be due to a distinct component of mastery. The endoscopist found that with experience came the ability to reduce the number of plications per procedure by increasing the distance between bites, without compromising the appearance of the endoscopic sleeve. This is notable because using less plications not only decreases the time necessary to complete the sleeve, but also decreases the cost.

Notably, only 7 procedures were required before the endoscopist reached $90 \%$ potential efficiency, following a 1-day train-

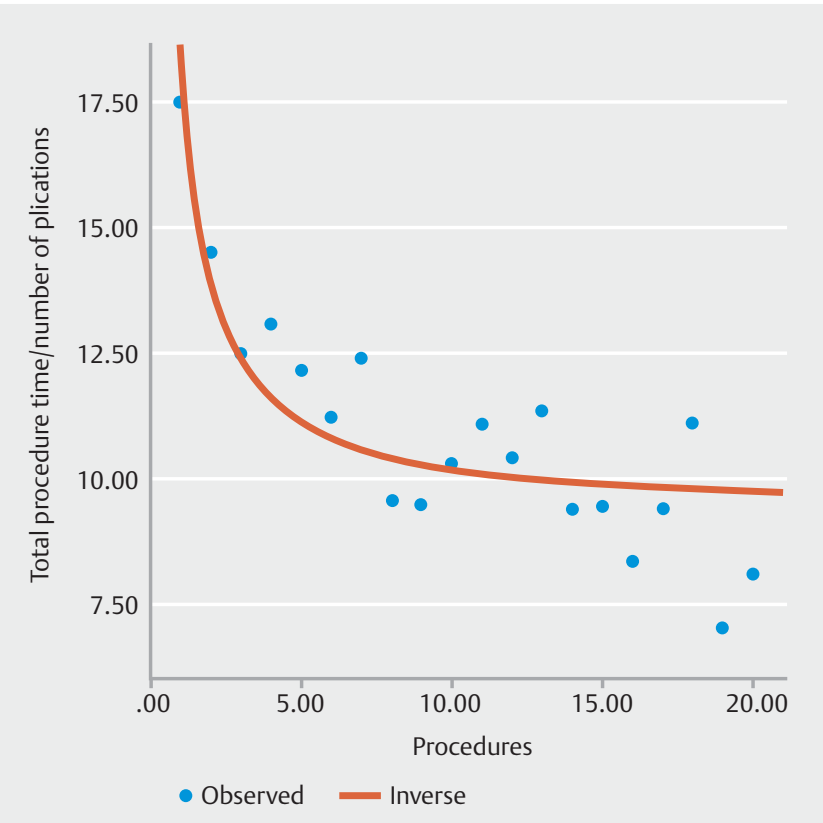

- Fig. 4 Total procedure time per number of plications decreased significantly over time, reaching a plateau at a rate of 9 procedures $(P<0.001)$.

ing session. This finding begins to fill a gap in our knowledge of ESG, and is an important factor to consider when deciding whether to incorporate ESG into one's treatment offerings. To effectively determine whether or not the investment in a procedure is worth the outcome, it is important to consider the learning curve in conjunction with the potential impact.

Weight loss reported post-ESG has been impressive, with multiple centers reporting over $50 \% \mathrm{EWL}$ at 1 year post-procedure $[1,5]$. Despite the suturing mechanism of ESG, the rate of AEs is minimal with a number of series reporting 0 events $[2,5]$. The recovery period is also short, with patients generally returning to full functionality within 1 to 3 days post-ESG [1]. Together, these factors contribute to the appeal and effectiveness of ESG, and therefore indicate the potential impact of the elective treatment. Given the results of this study, we hypothesize that the widespread implementation of this procedure will increase.

When interpreting the results of this learning curve analysis, it is essential to bear in mind the specific qualifications and experience of the performing endoscopist. The single-endoscopist modeled in this analysis is an expert in bariatric endoscopy who had experience in endoscopic suturing prior to training for ESG. It is also worth considering that all procedures in this analysis were performed with the assistance of nursing and endoscopy teams that were experienced in assisting endoscopic suturing, and which were held constant throughout the series. This factor likely has an effect on procedural times, but is not quantifiable. To perform ESG, the performing endoscopist must be able to place full-thickness bites using the OverStitch device, however, no specific duration of experience is required. 
The ESG procedure is repetitive in nature, which likely contributes to the speed of the learning curve.

Because the analysis was restricted to the learning curve of only 1 endoscopist, the results will likely vary when applied to a variety of endoscopists. This point is further evidenced in the variation of median and mean procedure times in the current literature, which ranges from 75 to 157 minutes, in series of 20 and 10 patients, respectively $[2,4,5]$. Currently, it is not possible to compare other reports of an ESG learning curve to this one, as there are no published reports to date. However, Abu Dayyeh et al. has previously indicated the presence of a learning curve in his series of cases. First, in a 4-case series, procedure time decreased significantly from 245 minutes in the initial patient to 172 minutes in the fourth, despite 3 additional sutures, therefore indicating a learning curve [3]. In a later study, Abu Dayyeh et al. determined that procedure times significantly decreased in a series of 25 cases, from $217 \pm 17$ minutes (average of first 5 procedures) to $98 \pm 4$ minutes (average of last five procedures) $(P<.01)[1]$.

It is worth noting that sample size is not a limitation of the study, as the plateau for all 3 variables was reached. Increasing the sample size is not predicted to alter the value at which each variable reached a plateau. Details of weight outcomes, as well as long-term outcomes, are not reported here so as to not double publish data. A more detailed analysis of the weight outcomes is reported in a separate, larger study. Data on suture stability at follow-up is not reported, because ESG is a self-pay procedure and we could not justify a second-look endoscopy or upper gastrointestinal endoscopy to assess suture strength at follow-up. When considering undergoing ESG training, it is important to consider the length of time between the training session and clinical ESG procedure. We recommend a short interval of time between the two. In this case, there was a 1-week interval between training and procedure. Whether the interval between training and procedure would significantly affect the learning curve is uncertain, but it is a factor worth considering.

\section{Conclusion}

We conclude that an endoscopist proficient in endoscopic suturing can expect to acquire efficiency in performing ESG after approximately 7 cases. Further, the number of necessary plications can be expected to decrease, with an expected 8 sutures per patient once proficiency has been reached. Therefore, the learning curve for ESG in endoscopists familiar with the OverStitch suturing device is relatively short and should encourage interested endoscopists to perform this procedure to help stem the obesity epidemic.

\section{Competing interests}

Dr. Khashab is a consultant for Boston Scientific and Olympus America and has received research support from Cook Medical. Dr. Kumbhari is a consultant for Boston Scientific and Apollo Endosurgery. Dr. Kalloo is a founding Member, equity holder, and consultant for Apollo Endosurgery.

\section{References}

[1] Abu Dayyeh Bk, Acosta A, Camilleri M et al. Endoscopic sleeve gastroplasty alters gastric physiology and induces loss of body weight in obese individuals. YJCGH Clin Gastroenterol Hepatol 2017; 15: 37 -43 .e31

[2] Sharaiha RZ, Kedia P, Kumta N et al. Initial experience with endoscopic sleeve gastroplasty: technical success and reproducibility in the bariatric population. Endoscopy 2015; 47: 164 - 166

[3] Abu Dayyeh BK, Rajan E, Gostout C]. Endoscopic sleeve gastroplasty: a potential endoscopic alternative to surgical sleeve gastrectomy for treatment of obesity. YMGE Gastrointest Endosc 2013; 78: 530-535

[4] Lopez-Nava G, Galvaão MP, da Bautista-Castaño I et al. Endoscopic sleeve gastroplasty for the treatment of obesity. Endoscopy 2015; 47: $449-452$

[5] Lopez-Nava G, Galvão M, Bautista-Castao I et al. Endoscopic sleeve gastroplasty with 1-year follow-up: factors predictive of success. Endoscop Int Open 2016; 4: 222-227

[6] Colquitt JL, Pickett K, Loveman E etal. Surgery for weight loss in adults. Cochrane Database Syst Rev 2014. doi:10.1002/14651858. CD003641.pub4

[7] Barola S, Agnihotri A, Khashab MA et al. Perigastric fluid collection after endoscopic sleeve gastroplasty. Endoscopy 2016; 48: E340E341

[8] Lopez-Nava G, Galvão MP, Bautista-Castaño I et al. Endoscopic sleeve gastroplasty: How I do it? Obes Surg 2015; 25: 1534 - 1538

[9] Barola S, Kalloo A, Khashab MA et al. Technical aspects of endoscopic sleeve gastroplasty. VideoGIE 2017; 2: 48

[10] Feldman LS, Cao J, Andalib A et al. A method to characterize the learning curve for performance of a fundamental laparoscopic simulator task: defining "learning plateau" and "learning rate". Surgery 2009; 146: $381-386$

[11] Cotton PB, Eisen GM, Aabakken L et al. A lexicon for endoscopic adverse events: report of an ASGE workshop. Gastrointest Endosc 2010; 71: $446-454$ 